\title{
Special value formulae for Rankin L-functions
}

\author{
V. Vatsal
}

August 12, 2003

\section{INTRODUCTION}

Let $F$ denote a totally real number field, and let $K / F$ denote a totally imaginary quadratic extension. We fix an automorphic cuspidal representation $\pi$ of $G L_{2}(F)$, and a finite order Hecke character $\chi$ of $K$. Thus $\chi$ is a representation of $G L_{1}(K)$.

Under certain hypotheses, it is known that the central critical value $L(\pi \otimes \chi, 1 / 2)$ is algebraic up to a known transcendental factor. Explicit formulae for this value have been given by a number of authors, notably Gross, Waldspurger, and Zhang. Essentially, the work of Gross and Zhang shows that this value is given by the height of a certain CM divisor on a suitable space, while the work of Waldspurger gives a criterion for nonvanishing of this value in terms of a certain linear functional arising from representation theory, and a formula in terms of torus integrals on a quaternion algebra. Our goal in this article is to explicate the connections between these works, and to provide a bridge between the general representation-theoretic framework described by Gross (see his article [Gro] in this volume) and the theorems of Zhang [Zhaora] and Waldspurger [Wal85].

We want to point out that the formula we will discuss has numerous applications to arithmetic and Iwasawa theory (see [BD96] and its various sequels). We will therefore attempt to formulate the representation-theoretic results in terms that are familiar to number theorists. We will not however discuss any arithmetic applications directly - the reader will find some of these applications elsewhere in this volume.

Needless to say, the present work is mostly expository. The ideas are largely drawn from the papers [Gro87], [Gro], [Wal85], [Zhao1a]. However, the organization here is perhaps novel. Our main contribution is given in Theorem 6.4. While the ingredients in this theo- 
rem are all well-known, our formulation seems to be new, and is well-suited for applications to number theory as in [BD96] and [Vato2]. I would like to thank Benedict Gross, ShouWu Zhang, and Hui Xue for patiently answering my numerous questions on this subject. I would also like to thank Barry Mazur, Christophe Cornut, and the mathematics department at Harvard University for their hospitality in April 2002.

\section{NotATION AND Hy POTHESES}

We start by briefly recalling the basic notions about modular forms in the adelic setting. For generalities on automorphic forms and representations, we refer the reader to [Bum97], Chapter 3, or to the article [BJ79]. A clear discussion may also be found at the end of [Cas73]. Recall that an automorphic form is a function of moderate growth on $G L_{2}(F) \backslash G L_{2}$ (A), fixed under right translations by some open compact subgroup of $G L_{2}\left(\mathbf{A}_{f}\right)$, smooth at infinity, and contained in some finite dimensional space which is invariant under both a maximal compact subgroup, and the center of the universal enveloping algebra, of the real group $G L_{2}\left(F_{\mathbf{R}}\right)$. There is a map from classical Hilbert modular forms to the space of automorphic forms, as in [BJ79], section 4.3. The group $G L_{2}$ (A) acts on the space of automorphic forms, with the conventions of [Bum97], section 3.3. In particular, the action of $G L_{2}\left(\mathbf{A}_{f}\right)$ is by right translations. The structure at infinity is more complicated, since the space of automorphic forms is not preserved by right translations by $G L_{2}\left(F_{R}\right)$. For a detailed discussion of the structure at infinity, we refer the reader to Bump's book.

Now let $\pi$ denote a cuspidal automorphic representation of $G L_{2}(\mathbf{A})$. Then $\pi$ may be viewed in (at least) two different ways. On the one hand, $\pi$ is by definition an irreducible subquotient of $\mathcal{A}$, where $\mathcal{A}$ is the space of automorphic cuspforms on $G L_{2}(\mathbf{A})$. On the other hand, it is a theorem of Flath that any such subquotient is abstractly isomorphic to a restricted tensor product $\pi \cong \otimes \pi_{v}$, where each $\pi_{v}$ is a representation of $G L_{2}\left(F_{v}\right)$, at least at the finite places. The components $\pi_{v}$ at real places $v$ are not quite representations of $G L_{2}(\mathbf{R})$; rather, they are just the $(\mathfrak{g}, K)$-modules of [Bum97], Chapter 2. We will need both descriptions of automorphic representations in the sequel. It is a fact that any cuspidal automorphic representation $\pi$ occurs with multiplicity one in the space of automorphic forms. To each automorphic representation $\pi$ is attached a certain integral ideal $N$ of $F$, called the conductor of $\pi$. Then $N$ depends only on the local components $\pi_{v}$. We will give the exact definition of $N$ 
in the next section, following [Cas73].

Next we need to recall some simple facts about Whittaker coefficients of automorphic forms, which are the adelic analogue of Fourier coefficients in the classical theory. We will also need to understand how Whittaker coefficients transform with respect to the Hecke operators. Thus fix a nontrivial additive character $\eta$ of $\hat{F} / F$. For any automorphic cuspform $\phi$ and an idele $a$ of $F$, define a function $W_{\phi}$ on $G L_{2}(\hat{F})$ by

$$
W_{\phi}(g)=\int_{\hat{F} / F} \phi\left(\left(\begin{array}{ll}
1 & x \\
0 & 1
\end{array}\right) g\right) \eta(-x) d x .
$$

Then $\phi$ has a 'Fourier expansion'

$$
\phi(g)=\sum_{\alpha \in F^{\times}} W_{\phi}\left(\left(\begin{array}{ll}
\alpha & 0 \\
0 & 1
\end{array}\right) g\right) .
$$

For a proof of the above fact, we refer the reader to [Bum97], Theorem 3.5.5. It follows from the Fourier expansion that $\phi$ is determined by the Whittaker function $W_{\phi}$. Furthermore, the strong approximation theorem implies that $W_{\phi}$ is in turn determined by the numbers

$$
W_{\phi}\left(\left(\begin{array}{ll}
a & 0 \\
0 & 1
\end{array}\right) g_{\infty}\right),
$$

for $a \in \hat{F}_{f}^{\times}$and $g_{\infty} \in G L_{2}\left(F_{\mathbf{R}}\right)$. We call these numbers the Whittaker coefficients of $\phi$.

The Whittaker coefficients satisfy a simple transformation rule under the action of the Hecke operators $T_{v}$. To state this, let $v$ denote any finite place of $F$, relatively prime to the level of $\phi$. Then one can define a Hecke operator $T_{v}$ acting on $\phi$. (For the precise definition, we refer to [Bum97], Chapter 4, or equation (15) below.) Then $T_{\nu} \phi$ is also a cuspform, and we have

$$
W_{\phi}\left(\left(\begin{array}{cc}
a \varpi_{v} & 0 \\
0 & 1
\end{array}\right) g_{\infty}\right)=\left|\varpi_{v}\right| W_{T_{\nu} \phi}\left(\left(\begin{array}{ll}
a & 0 \\
0 & 1
\end{array}\right) g_{\infty}\right) .
$$

Here $\varpi$ denotes a local uniformizer at $v$. We will use this formula later.

Finally, we want to point out that the classical Petersson inner product on Hilbert modular forms has an analog from the adelic point of view. Indeed, it can be shown that if $\phi$ is any automorphic cuspform on $G L_{2}(\mathbf{A})$, such that the center $Z(\mathbf{A})$ acts on $\phi$ via a unitary 
character, then $\phi$ is square integrable modulo $Z(\mathbf{A}) G L_{2}(F)$. For this we refer the reader to [BJ79], section 4.4. Thus we may define an inner product pairing on the space of cuspforms by

$$
\left(\phi_{1}, \phi_{2}\right)=\int_{Z(\mathbf{A}) G L_{2}(F) \backslash G L_{2}(\mathbf{A})} \phi_{1}(g) \bar{\phi}_{2}(g) d g,
$$

where $d g$ denotes any Haar measure. In practice, one must normalize the measure depending on the application in view. Typically, one requires that some fixed open compact subgroup $U$ gets measure 1. We will attempt to be careful about this in the exact formulae later in this article.

Now we specialize everything to the representations $\pi$ and $\chi$ of interest. Thus recall that $\pi$ is a representation of $G L_{2}(F)$, and the finite order Hecke character $\chi$ is a representation of $G L_{1}(K)$. We will impose the following basic assumptions and notations which will be in force throughout this paper.

1. If $v$ is any infinite place of $F$, then the local component $\pi_{v}$ of $\pi$ at $v$ is a weight two discrete series representation.

2. The central character of $\pi$ is trivial. (This means that the center $Z(\mathbf{A})$ acts trivially.)

3. The conjugate of $\chi$ under the action of $\mathrm{Gal}(K / F)$ is equal to $\chi^{-1}$ and $\chi$ is trivial on $G L_{1}(F)$.

4. If $N$ denotes the conductor of $\pi$, and $d$ denotes the relative discriminant of $K / F$, then $(N, d)=1$. Here $N$ and $d$ denote integral ideals of the ring of integers $O_{F}$ of $F$.

5. If $c$ denotes the conductor of $\chi$, then $(c, N d)=1$. Note that it follows from assumption 3 above that the conductor of $\chi$ is invariant under $\operatorname{Gal}(K / F)$ and so may be identified with an ideal of $F$.

More concretely, we assume that $\pi$ corresponds to a holomorphic Hilbert modular form of weight $(2, \ldots 2)$, with trivial central character, and that the character $\chi$ is anticyclotomic. Some of the hypotheses above may be weakened, but for the sake of clarity, it is convenient to impose the extra conditions. We set $D=d c^{2}$.

Now consider the representation $\pi \otimes \chi$ of $G L_{2}(F) \otimes G L_{1}(K)$. Let $L(\pi \otimes \chi, s)$ denote the associated L-function. Then $L(\pi \otimes \chi, s)$ has a functional equation of the form $L(\pi \otimes$ 
$\chi, s)=\epsilon(\pi \otimes \chi, s) L(\pi \otimes \chi, 1-s)$. For a detailed discussion of the representation $\pi \otimes \chi$, we refer to the article of Gross in this volume. The L-function and its functional equation are discussed in Chapter 2 of [Zhaoia]. Under the hypotheses on $\pi$ and $\chi$ stated above, it can be shown that

$$
\epsilon(\pi \otimes \chi, 1 / 2)=(-1)^{\# \Sigma}
$$

where $\Sigma$ denotes the set of infinite places of $F$, together with the set of finite places $v$ such that $\omega_{v}(N)=-1$. Here $\omega$ denotes the quadratic character of the ideles $\hat{F^{\times}}$of $F$, defined by the extension $F / K$, and $\omega_{v}$ denotes the local component. In other words, $\Sigma$ consists of the infinite places together with finite places $v$ such that $v$ is inert in $K$, and such that $\operatorname{ord}_{v}(N)$ is odd. We shall say that we are in the definite case if $\Sigma$ is even, and in the indefinite case if $\Sigma$ is odd. Observe that, under the present hypotheses, the cardinality of $\Sigma$ depends only on $\pi$ and $K$, and not on the character $\chi$. A general formula for $\epsilon(\pi \otimes \chi, s)$ may be found in Chapter 3 of [Zhao1a], especially equation (3.1.2).

Evidently, we have $L(\pi \otimes \chi, 1 / 2)=0$ if we are in the indefinite case. This is the case originally treated by Gross and Zagier, and subsequently generalized by Zhang [Zhaora], [Zhaorb]. We shall not consider this case here. Rather, we will concentrate on explicating the formulae for the value $L(\pi \otimes \chi, 1 / 2)$ given in the definite case by Waldspurger [Wal85], Gross [Gro87], and Zhang [Zhaora]. Thus, from now on, we assume that we are in the definite case, so that the set $\Sigma$ has even cardinality.

\section{ATKIn-LEHNER THEORY ON $G L_{2}$}

Now we want to discuss newforms in the adelic setting. First recall classical Atkin-Lehner theory for modular forms on congruence subgroups of $S L_{2}(\mathbf{Z})$. The basic result states that each cuspform $g$ of level $M$ which is an eigenform for almost all the Hecke operators is given by $g(z)=\sum_{a} c_{a} g_{0}(a z)$, where $g_{0}$ is a unique form of some level $N \mid M$, which is an eigenform for all the Hecke operators at level $N$, and $a$ runs over divisors of $M / N$. The form $g_{0}$ is called the Atkin-Lehner newform; it depends only on the package of eigenvalues attached to $g$.

We want an analog of this theorem in the adelic set-up. Casselman's beautiful idea is to construct a newform $\phi_{v}$ locally in each representation $\pi_{v}$. Then the global newform is just the tensor product $\otimes \phi_{v}$, as $v$ runs over all places. Note however that this produces the newform as an abstract vector in the restricted tensor product $\pi=\otimes \pi_{v}$; to obtain a genuine 
automorphic form, one must embed $\pi$ in the space of functions $\mathcal{A}$ as above.

To describe the construction, let $v$ denote a finite place of $F$, and let $\varpi$ denote a local uniformizer at $v$. For a non-negative integer $c$, we define a group $U_{0}\left(\varpi^{c}\right)$ by putting

$$
U_{0}\left(\varpi^{c}\right)=\left\{\gamma \in G L_{2}\left(O_{F, v}\right): \gamma \equiv\left(\begin{array}{cc}
* & * \\
0 & *
\end{array}\right) \quad\left(\bmod \varpi^{c}\right) .\right\}
$$

Let $\pi_{v}$ denote the local component of $\pi$ at $v$. Then Casselman proves the following theorem.

Theorem 3.1 Let $c=c_{v}$ denote the smallest non-negative integer such that $U_{0}\left(\varpi^{c}\right)$ has a nonzero fixed vector in $\pi_{v}$. Then the fixed space of $U_{0}\left(\varpi^{c}\right)$ in $\pi_{v}$ has dimension 1.

Definition 3.2 The ideal $N_{v} \subset O_{F, v}$ generated by $\varpi_{v}^{c_{v}}$ is called the conductor of $\pi_{v}$. The conductor of $\pi$ is the ideal $N=\prod_{v} N_{v}$.

It can be shown that $c_{v}=0$ for almost every $v$. A nonzero vector $\phi_{v}$ fixed by $U_{0}\left(\varpi^{c_{v}}\right)$ is called a local newform at $v$. Note that $\phi_{v}$ is fixed by $G L_{2}\left(O_{F, v}\right)$ for almost all $v$. In this case, we say that $\pi$ is unramified at $v$.

There is a corresponding statement at the archimedean places of $F$. However, matters are somewhat more complicated, since, as we have already remarked, the local factor at infinity is not a local representation. We will not enter into a discussion of this point here. Suffice it to say simply that the nature of the infinite component is given by the weight, which in our case is $(2, \ldots, 2)$. Again, there exists a local newform $\phi_{v}$ for each $v \mid \infty$.

Definition 3.3 The Atkin-Lehner newspace is the line in $\pi=\otimes \pi_{v}$ spanned by $\otimes \phi_{v}$, where, for each $v, \phi_{v}$ is a local newform at $v$.

Thus an Atkin-Lehner newform for $\pi$ is a nonzero vector in the newspace. It is fixed by the group $U_{0}(N)=\left\{\gamma \in G L_{2}\left(\hat{O}_{F}\right): \gamma \equiv\left(\begin{array}{ll}* & * \\ 0 & *\end{array}\right)(\bmod N)\right\}$. As such, it is defined only up to scalars.

To state the main result of Atkin-Lehner theory, we first need to introduce notation. Let $\pi$ be given, and fix a newform $\phi$ for $\pi$. Let $a$ denote any ideal of $F$ which is relatively prime to 
the level. We may identify $a$ with an idele of $F$ in the usual way. We let

$$
g_{a}=\left(\begin{array}{cc}
a^{-1} & 0 \\
0 & 1
\end{array}\right),
$$

and define the function $\phi_{a}$ by $\phi_{a}(x)=\phi\left(x g_{a}\right)$. This is well-defined independent of the idele representing the ideal $a$, since $(a, N)=1$, and $\phi_{v}$ is fixed by $G L_{2}\left(O_{F, v}\right)$.

Now let $D$ denote any ideal prime to $N$, and write $V(\pi, N D)$ for the finite dimensional space of vectors in $\pi$ fixed by the group $U_{0}(N D)$. We call $V(\pi, N D)$ the space of vectors of level ND.

Theorem 3.4 ([CAs73]) The space $V(\pi, N D)$ has a basis consisting of the vectors $\phi_{a}$, as a runs over divisors of $D$.

Finally, we need to say a word about normalization of the Atkin-Lehner newform, since it is only defined up to scalars. In the classical theory, it is customary to normalize a newform in terms of the Fourier expansion, by requiring that a normalized newform have first coefficient equal to 1. In the adelic situation, we normalize via Whittaker functions, as in [Bum97], or [Zhao1a], section 2.5. The details are somewhat technical and we will not reproduce them here. Essentially, one starts by realizing the newform as function on the adeles. Then the corresponding Whittaker function $W_{\phi}$ breaks up as a tensor product of local Whittaker functions $W_{\phi, v}$, and we normalize each $W_{\phi, v}$ so that it takes the value 1 at the identity element, at least at good primes, and if the conductor of the additive character is 1 . In general, one can describe the normalization by requiring that the Mellin transform of the local Whittaker function $W_{\phi, v}$ be equal to the local L-function $L\left(s, \pi_{v}\right)$. For discussion of the normalization we refer to Zhang's paper [Zhaoia].

\section{QUATERNION ALGEBRAS AND THE JACQUET-LANGLANDS CORRESPONDENCE}

We want to transfer the representation $\pi$ of $G L_{2}$ to a representation $\pi^{\prime}$ of a suitable quaternion algebra $B$. Indeed, we may take for $B$ the unique quaternion algebra ramified precisely at the set of primes in $\Sigma$, which has even cardinality. Furthermore, since $\Sigma$ contains all the infinite places, we see that the algebra $B$ is totally definite. Since each $v \in \Sigma$ is such that $\pi_{v}$ is special or supercuspidal if $v$ is finite, and in the discrete series if $v$ is infinite, we see that $\pi_{v}$ is squareintegrable for every $v \in \Sigma$. Thus the Jacquet-Langlands correspondence implies that a lift $\pi^{\prime}$ 
of $\pi$ to $B$ exists. Furthermore, there exists an embedding $K \rightarrow B$ (since every prime $v \in \Sigma$ is by definition inert in $K$ ). We fix such an embedding once and for all. We remark here that a very readable summary of the Jacquet-Langlands correspondence may be found in the book [Lub94]

Now let $R=\prod R_{v}$ denote an order of $\hat{B}=B(\hat{F})$ defined by requiring that $R_{v} \subset B_{v}$ be an order of reduced discriminant $N_{v}$ which optimally contains $O_{K, v}$. For a proof that such orders exist, we refer to [Gro88], Proposition 3.6. Here $N$ is the level of $\pi$ as before, and $N_{v}$ denotes the local component at $v$.

We put $U_{v}=R_{v}^{\times}$, so that $U=\prod U_{v}$ is an open compact subgroup of $\hat{B}^{\times}$. Then it follows from work of Gross and Prasad [GP91], Propositions 2.3 and 2.6, that the subgroup $U_{v}$ fixes a unique line $L_{v}$ in the representation space $\pi_{v}$ for each $v$. We fix a nonzero vector $\psi_{v} \in L_{v}$ for each $v$, and write $\psi=\otimes \psi_{v} \in \pi$, where we have decomposed $\pi$ as a restricted tensor product relative to the subgroups $U_{v}$. This makes sense because $U_{v}=R_{v}^{\times}$is a maximal compact subgroup of $B_{v}$ for almost every $v$. We may regard $\psi$ as the analog of an Atkin-Lehner newform for the representation $\pi^{\prime}$.

It is known that there is a realization of $\pi^{\prime}$ in the space $\mathcal{S}$ of cuspforms on $B(F)^{\times} \backslash \hat{B}^{\times}$, where the action of $\hat{B}^{\times}$is by right translations. Indeed, $\pi^{\prime}$ occurs with multiplicity one in $\mathcal{S}$. We fix an embedding $\pi \rightarrow \mathcal{S}$. Note that such an embedding is defined only up to scaling. From this viewpoint, $\psi$ may be considered as a locally constant function on $\hat{B}^{\times}$, left invariant under $B(F)^{\times}$, and right invariant under $\hat{F}^{\times} \cdot U$, where the invariance under $\hat{F}^{\times}$comes from our assumption that $\pi$ and $\pi^{\prime}$ have trivial central character. Note also here that since $\pi$ has weight $(2, \ldots, 2)$ and $B$ is totally definite at infinity, the Jacquet-Langlands correspondence implies that the infinite component of $\pi^{\prime}$ is just the trivial representation of the compact group $B\left(F_{\mathbf{R}}\right)^{\times} / F_{\mathbf{R}}^{\times}$

\section{THE WORK OF WALDSPURger}

Now we fix an anticyclotomic character $\chi$ of $G L_{1}(K)$. We retain the hypotheses on $N$ and $D$ made in the previous sections, and, as before, $c$ will denote the conductor of $\chi$. We identify the quadratic extension $K / F$ with a maximal torus $T$ of $B^{\times}$, and consider a realization of $\pi^{\prime}$ in the space of functions on $\hat{B}^{\times}$. The character $\chi$ of $G L_{1}(\hat{K})$ is a homomorphism $\hat{K}^{\times} / K^{\times} \rightarrow$ $\mathbf{C}^{\times}$, and we write $\mathbf{C}(\chi)$ to denote the associated representation of $G L_{1}(\hat{K})$. If $v$ is any place 
of $F$, we will use a subscript $v$ to denote the corresponding local object.

With these notations, the fundamental local result is the following proposition of Waldspurger [Wal85] and Tunnell [Tun83].

Proposition 5.1 Let $v$ denote any place of F. Put $V\left(\pi_{v}, T_{v}, \chi_{v}\right)=\operatorname{Hom}_{T_{v}}\left(\pi_{v}, \mathbf{C}\left(\chi_{v}\right)\right)$. Then $\operatorname{dim}_{\mathrm{C}}\left(V\left(\pi_{v}, T_{v}, \chi_{v}\right)\right)=1$.

For a discussion of this result, and connection with local root numbers of $\pi, \pi^{\prime}$, and $\chi^{\prime}$, we refer the reader to Gross' article in this volume.

It follows from the local result above that $V(\pi, T, \chi)=\operatorname{Hom}_{T}(\pi, \mathbf{C}(\chi))$ has dimension 1. Furthermore, it is easy to exhibit a candidate for an element of this one-dimensional space. Indeed, it is clear that the functional defined by

$$
e \mapsto \ell(e)=\int_{T(F)^{\times} \hat{F}^{\times} \backslash \hat{T}^{\times}} e(t) \chi^{-1}(t) d t
$$

is an element (possibly zero) of $\operatorname{Hom}_{T}(\pi, \mathbf{C}(\chi)$ ). Here we may take $d t$ to denote any Haar

measure on $\hat{T}$, since any two such differ only by a constant multiple. Note also that the integral converges because the domain of integration is compact.

With this notation, Waldspurger proved the following global result (see [Wal85], Théorème 2, page 221).

Theorem 5.2 The functional $\ell$ is nonzero on $\pi^{\prime}$ if and only if $L(\pi \otimes \chi, 1 / 2) \neq 0$.

The above theorem may be viewed as giving a criterion for the nonvanishing of $L(\pi \otimes$ $\chi, 1 / 2)$. Namely, to show that $L(\pi \otimes \chi, 1 / 2)$ is nonzero, it suffices to exhibit an element $e \in \pi^{\prime}$ such that $\ell(e)$ is nonzero. Note, however, that the linear form $\ell$ and the 'test' vector $e$ are defined only up to scalar, and that there is no obvious way (yet) to recover the actual value $L(\pi \otimes \chi, 1 / 2)$. We will return to this question later.

\section{Test vectors: the work of Gross and Prasad}

In this section, we will review the basic results of [GP91], where the problem of constructing local test vectors is solved. Thus let $v$ denote any finite place of $F$ and let $\pi_{v}$ denote the local component of $\pi$ at $v$. Recall that we have fixed an embedding $K \rightarrow B$ and so an embedding $K_{v} \rightarrow B_{v}$. According to our hypotheses on $N$ and $D$, at least one of $\pi$ and $\chi$ is unramified 
at $v$. In each case, we wish to construct an explicit vector $\psi_{\chi, v} \in \pi_{v}$ such that $\ell_{v}\left(\psi_{\chi, v}\right) \neq 0$, where $\ell_{v}$ is any nonzero linear functional in $V\left(\pi_{v}, T_{v}, \chi_{v}\right)=\operatorname{Hom}_{T_{v}}\left(\pi_{v}, \mathbf{C}\left(\chi_{v}\right)\right)$.

First consider the case where $\pi_{v}$ is an unramified principal series representation. Then we see that $B_{v} \cong G L_{2}$. Let $c\left(\chi_{v}\right)$ denote the conductor of the character $\chi_{v}$ of $K_{v}$, and let $R_{v} \subset B_{v}$ denote a maximal order which optimally contains the order of $K_{v}$ with conductor $c\left(\chi_{v}\right)$. Then Gross and Prasad have shown that the group $R_{v}^{\times}$fixes a unique line in $\pi_{v}$. In this case, we let $\psi_{\chi, v}$ denote any nonzero vector fixed by $R_{v}^{\times}$.

Now suppose that $\pi_{v}$ is ramified, so that $\chi_{v}$ is unramified. In this case, let $R_{v} \subset B_{v}$ denote an order of reduced discriminant $N_{v}$ which contains $O_{K, v}$. (That such orders exist and are unique up to conjugation by $K_{v}$ is proved in [Gro88].) Again, the group $R_{v}^{\times}$fixes a unique line in $\pi_{v}$, and we let $\psi_{\chi, v}$ denote any nonzero vector on this distinguished line.

The following result restates Propositions 2.3 and 2.6 of [GP91].

Proposition 6.1 (Gross-Prasad) Let $\psi_{\chi, v}$ be defined as above. Then, if $\ell_{v}$ is any nonzero element of $V\left(\pi_{v}, T_{v}, \chi_{v}\right)$, we have $\ell_{v}\left(\psi_{\chi, v}\right) \neq 0$.

Now we want a global test vector. Recall that we have defined a 'newform' $\psi$ in section 4 above by specifying that $\psi=\otimes \psi_{v}$, where each $\psi_{v}$ is fixed by $R_{v}^{\times}$, for a suitable order $R_{v} \subset$ $B_{v}$. We will produce our test vector by modifying $\psi$ at places $v$ dividing $c=c(\chi)$. Indeed, it follows from the definitions that $\psi_{v}=\psi_{\chi, v}$ for almost all $v$. Indeed, this equality holds for all $v \nmid c(\chi)$. Thus we may consider the vector $\psi_{\chi}=\otimes \psi_{\chi, v}$ as an element of the restricted tensor product $\otimes \pi_{v}$. Then the following proposition may be extracted from [Wal85], and resolves the question of global test vectors:

Proposition 6.2 Let $\psi_{\chi}$ be defined as above. Let $\ell \in \operatorname{Hom}_{T}\left(\pi^{\prime}, \mathbf{C}(\chi)\right)$ denote the functional defined in equation (3). Then we have $\ell \neq 0$ if and only if $\ell\left(\psi_{\chi}\right) \neq 0$. In particular, $L(\pi \otimes$ $\chi, 1 / 2) \neq 0$ if and only if $\ell\left(\psi_{\chi}\right) \neq 0$.

Our next task is to produce a formula for the number $\ell\left(\psi_{\chi}\right)$. According to the definitions, we have

$$
\ell\left(\psi_{\chi}\right)=\int_{T(F)^{\times} \hat{F}^{\times} \backslash \hat{T}^{\times}} \psi_{\chi}(t) \chi^{-1}(t) d t .
$$

Now observe that the function $\psi_{\chi}$ is invariant on the right by the group $\prod R_{v}^{\times}$, where $R_{v}$ is an order of $B_{v}$ which optimally contains the order of $O_{K, v}$ with conductor $c_{v}=c\left(\chi_{v}\right)$. Since $\chi$ 
is invariant under $\hat{O}_{c}^{\times}$, it follows that the integral in (4) may be rewritten as a finite sum:

$$
\ell\left(\psi_{\chi}\right)=\mu_{\chi} \cdot \sum_{t \in G_{c}} \psi_{\chi}(t) \chi^{-1}(t)
$$

where $G_{c}$ denotes the finite set $T(F)^{\times} \hat{F}^{\times} \backslash \hat{T}^{\times} / \hat{O}_{c}^{\times}$, and $\mu_{\chi}$ is the volume of the image of $\hat{O}_{c}^{\times}$ in $T(F)^{\times} \hat{F}^{\times} \backslash \hat{T}^{\times}$. Observe that, by class field theory, we may identify $G_{c}$ with the quotient of $\operatorname{Pic}\left(O_{c}\right)$ by $\operatorname{Pic}\left(O_{F}\right)$.

One can even go slightly further, and express the right-hand-side of (5) in terms of the newform $\psi$ (which is independent of $\chi$ ). As we will see, this leads naturally to the appearance of certain CM points of conductor $c(\chi)$.

To begin with, recall that $\psi$ and $\psi_{\chi}$ agree at all places $v$ except those finite places which divide $c$. If $v$ divides $c$, then $B_{v} \cong G L_{2}\left(F_{v}\right)$ is split, and $\psi_{v}$ and $\psi_{\chi, v}$ are fixed by maximal orders $R_{v}$ and $R_{\chi, v}$ respectively, where $R_{v} \cap K_{v}=O_{K, v}$ and $R_{v, \chi} \cap K_{v}=O_{\chi, v}$ is the order of conductor $c_{v}$. Since all maximal orders in $B_{v}$ are conjugate, it follows that $\psi_{\chi, v}$ and $\psi_{v}$ are related by the equation $\psi_{\chi, v}(z)=\psi_{v}\left(z g_{v}\right)$, where $g_{v}$ is such that $g_{v} R_{v} g_{v}^{-1}=R_{\chi, v}$. Thus, if we let $g \in \hat{B}$ denote an element such that $\psi_{\chi}(z)=\psi(z g)$, the sum in equation (5) becomes $\sum_{t \in G_{c}} \psi(t g) \chi^{-1}(t)$.

Now observe that, for each place $v$ of $F$, the order $t_{v} g_{v} R_{v}\left(t_{v} g_{v}\right)^{-1}$ is another local order of $B_{v}$ which optimally contains $O_{\chi, v}$. For each $t$, let $R_{t}$ denote the global order of $B$ defined by $R_{t}=B \cap \operatorname{tg} \hat{R}(\operatorname{tg})^{-1}$. Then $R_{t}$ is an order with discriminant $N$ which optimally contains $O_{c} \subset O_{K}$. Furthermore, it is clear that if $R^{\prime}$ is any order of $B$ with discriminant $N$ which optimally contains $O_{c}$, then $R^{\prime}=R_{t}$ for some $t$. (Here we remind the reader that the embedding $K \rightarrow B$ is fixed.)

Note that the function $\psi$ factors through the coset space $\operatorname{Cl}(B)=\operatorname{Cl}(B, N)=B^{\times} \backslash \hat{B}^{\times} / \hat{F}^{\times} \hat{R}^{\times}$. The set $\mathrm{Cl}(B)$ may be identified with conjugacy classes of oriented orders of discriminant $N$ in $B$. If $R^{\prime}$ is any such order, then $R^{\prime}$ determines an element of $\mathrm{Cl}(B)$, and thus it makes sense to speak of the value $\psi(B)$. From this viewpoint, we see that the sum in (5) is just $\sum_{t} \chi^{-1}(t) \psi\left(R_{t}\right)$, and that the set $R_{t}$ runs over oriented orders of $B$ of discriminant $N$ which optimally contain $O_{c}$. 


\section{CM points}

The reader who is familiar with the formalism of [BD96] and [Gro87] will recognize the optimal embeddings $O_{c} \rightarrow R_{t}$ occurring in the above as being precisely the points called 'definite' Heegner points, or CM points, in the former and special points in the latter. We now proceed to describe these special points from a more adelic point of view, and rewrite Waldspurger's theorem in terms of an evaluation of $\psi$ on a suitable CM cycle.

Thus let $G^{\prime}$ denote the algebraic group $B^{\times} / F^{\times}$. Let $U$ denote any open compact subgroup of $G$. Recall that we have fixed an embedding $K \rightarrow B$, and let $T$ denote the torus $K^{\times} / F^{\times} \subset$ $G$. Then the set of CM points of level $U$ on $B$ associated to the embedding $K \rightarrow B$ is defined to be the coset space

$$
C=T(F) \backslash G^{\prime}\left(\mathbf{A}_{f}\right) / U,
$$

where $\mathbf{A}_{f}$ denotes the space of finite adeles. Note that there is an action of $\hat{T} / T(F)=$ $\hat{K}^{\times} / K^{\times} \hat{F}^{\times}$on the set $C$. A $C M$ cycle is just a compactly supported function on $C$. In other words, a CM cycle is just a finite linear combination of characteristic functions of cosets in $C$.

We can make this definition more concrete in the case that $U$ is the image of $\hat{R}^{\times}$, for some order $R \subset B$ of discriminant $N$. Indeed, in that case, each element $P$ in $C$ is represented by some $x \in \hat{B}$, and we may form the order $B_{x} \subset R$ defined by $B_{x}=B \cap x \hat{R} x^{-1}$. Let $O_{x} \subset O_{K}$ denote the order given by $O_{x}=K \cap B_{x}$. By definition, the fixed embedding $f: K \rightarrow B$ induces an optimal embedding $O_{x} \rightarrow B_{x}$. Thus the choice of $x$ yields a pair $\left(O_{x}, R_{x}\right)$, where $f: O_{x} \rightarrow R_{x}$ is an optimal embedding. One checks furthermore that choice of a different representative $x^{\prime}$ for the coset defining the CM point $P$ yields a pair $\left(O_{x^{\prime}}, R_{x^{\prime}}\right)$ which differs from $\left(O_{x}, R_{x}\right)$ simply by conjugation by an element of $T$. The conductor of $P$ is defined to be the conductor $c$ of the order $O_{x}$, which is obviously independent of the choice of $x$. If $P$ has conductor $c$, then the action of $\hat{T} / T(F)$ on $P$ factors through $G_{c}$, where $G_{c}=\hat{K}^{\times} / \hat{F}^{\times} K^{\times} \hat{O}_{c}^{\times}$ as above. Furthermore, it is clear that $G_{c}$ acts simply transitively on the set of CM points of conductor $c$. If $\sigma \in G_{c}$ and $P$ is a CM point of conductor $c$, we write $P^{\sigma}$ to denote the image of $P$ under $\sigma$.

Remark 6.3 Suppose that $F=\mathbf{Q}$. Then a CM point of conductor $c$ is defined in [BD96] to be a pair $(f, R)$ where $R$ is an oriented order in $B$ of discriminant $N$, and $f: O_{c} \rightarrow R$ is an oriented embedding, where points $(f, R)$ and $\left(f^{\prime}, R^{\prime}\right)$ are identified if they are conjugate under the action of $B^{\times}$. One checks readily that this notion is equivalent to the one above. 
We can now combine Waldspurger's result with those of Gross-Prasad to obtain a simple criterion in terms of CM points for the non-vanishing of $L(\pi \otimes \chi, 1 / 2)$.

Theorem 6.4 Let the hypotheses and notation be as in section 2. Let $R$ denote any order of $B$ of discriminant $N$ which optimally contains $O_{K}$. Then, if $P$ is any $C M$ point of conductor $c$ and level $U=\hat{R}^{\times}$, we have $L(\pi \otimes \chi, 1 / 2) \neq 0$ if and only if $\sum_{\sigma \in G_{c}} \chi^{-1}(\sigma) \psi\left(P^{\sigma}\right) \neq 0$.

The theorem above seems to fill a gap in the literature, and is extremely convenient for applications to Iwasawa theory and arithmetic. Indeed, it is freely used in [BD97] and its various sequels, as well as in [Vato2]. (Note also that if $F=\mathbf{Q}$ and $K$ is an imaginary quadratic field, then $G_{c}=\operatorname{Pic}\left(O_{c}\right)$ since $\mathbf{Q}$ has class number 1.)

\section{The WORK OF Gross ANd ZHANG}

It is natural now to ask for an exact relationship between the numbers $L(\pi \otimes \chi, 1 / 2)$ and $\ell\left(\psi_{\chi}\right)$. More generally, one could ask for a relationship between $L(\pi \otimes \chi, 1 / 2)$ and the number $\sum_{\sigma \in G_{c}} \chi^{-1}(\sigma) \psi\left(P^{\sigma}\right)$ appearing in Theorem 6.4. These problems fall in the general category of Gross-Zagier formulae, in as much as they seek to express L-values in terms of explicit CM cycles. This problem was first taken up by Gross in [Gro87], where the case where $F=\mathbf{Q}$ and $N$ and $D$ are prime was treated. It was subsequently generalized to slightly more general $D$ in [Dag96]. The case of general $F$ and $D$ was finally treated in [Zhaora]. All of these results are more arithmetic in flavour than those of Waldspurger discussed above, being based on the calculation of Fourier coefficients of certain kernel functions for Rankin-Selberg convolutions, and the expression of these Fourier coefficients in terms of a height pairing on the CM cycles.

\section{Gross' formula}

To fix the ideas, we want to discuss the main result of [Gro87], which, as described above, deals with the case where $F=\mathbf{Q}$, and $N$ and $D$ are prime. For the benefit of the number theorists in the audience, we will start by describing the basic idea in classical rather than adelic language.

Thus let $N$ denote a positive rational integer, and let $g(z)=\sum a_{n} q^{n}$ denote a cuspform of weight 2 on $\Gamma_{0}(N)$. We normalize $g$ so that $a_{1}=1$. Let $K / \mathbf{Q}$ denote an imaginary quadratic 
field of discriminant $D$. We assume that both $N$ and $D$ are prime, and that $N$ remains inert in $K$. Let $\chi$ denote an unramified Hecke character of $K$, so that $c=1$. (Thus the notation for $N$ and $D$ employed here is consistent with the general case set out above.) Then $g$ corresponds to a cuspidal automorphic representation $\pi$ of $G L_{2}(\mathbf{Q})$, and $\chi$ is a representation of $G L_{1}(K)$. We want to study the value $L(\pi \otimes \chi, 1 / 2)$, which in classical notation (see [Maz84] for an overview) is just $L(g \otimes \chi, 1)$. Note that the normalization of the classical L-function $L(g \otimes$ $\chi, s)$ yields a functional equation under $s \mapsto 2-s$, while the automorphic L-function is symmetric under $s \mapsto 1-s$. As we have remarked, we will try to keep the classical notation in this section.

The starting point is the expression of $L(g \otimes \chi, s)$ as a Rankin-Selberg convolution. It is not our purpose here to discuss the Rankin-Selberg method in detail, so we will simply extract the one statement that is central to our discussion. The details may be found in [Gro87]. We recall that if $\Gamma$ is a congruence subgroup of $S L_{2}(\mathbf{Z})$ and $f, g$ are weight 2 modular forms with respect to $\Gamma$, then the Petersson inner product $(f, g)$ relative to $\Gamma$ is defined by

$$
(f, g)_{\Gamma}=8 \pi^{2} \int_{F_{\Gamma}} f(z) \bar{g}(z) d x d y
$$

where $F_{\Gamma}$ is a fundamental domain for $\Gamma$ in the upper half-plane, and $z=x+i y$. The integral converges provided that at least one of $f$ and $g$ is cuspidal.

Proposition 7.1 There exists a kernel function $\Theta_{\chi}$, which is a modular form of level ND, such that

$$
L(g \otimes \chi, 1)=\left(g, \Theta_{\chi}\right)_{N D}
$$

Here the Petersson product $(\bullet, \bullet)_{N D}$ is taken relative to the group $\Gamma_{0}(N D)$.

The first step in the argument is to take the trace of $\Theta_{\chi}$ down to level $N$. Thus put $\Theta=$ $\operatorname{Tr}_{N}^{N D}\left(\Theta_{\chi}\right)$. Then we clearly have

$$
L(g \otimes \chi, 1)=(g, \Theta)_{N},
$$

where this time the inner product is taken at level $N$. Now, $\Theta$ is a modular form of level $N$, so we may write

$$
\Theta=E(z)+\sum_{i} c_{i} g_{i}(z)
$$

where $E(z)$ is an Eisenstein series, and the sum is taken over a basis of the space of newforms of level $N$. (Since $N$ is prime. and we are in weight 2 , there are no oldforms.) We may assume 
that the numbering is such that $g(z)=g_{1}$. It can be shown that the coefficients $c_{i}$ are all real and algebraic, see [Shi76].

Then, by orthogonality, it is clear that

$$
(g, \Theta)_{N}=\left(g, E+\sum_{i} c_{i} g_{i}\right)_{N}=\left(g, c_{1} g_{1}\right)=c_{g}(g, g)
$$

where $c_{g}=c_{1}$ is the coefficient of $g$. Given an eigenform $g_{i}$, let us put $\Theta_{g_{i}}=c_{i} g_{i}$. We call $\Theta_{g_{i}}$ the $g_{i}$-isotypic component of $\Theta$. Thus $\Theta_{g_{i}}$ denotes the projection of $\Theta$ to the eigenspace of the Hecke algebra with eigenvalues given by the newform $g_{i}$. With this notation, we have

$$
L(g \otimes \chi, 1)=(g, \Theta)_{N}=\left(g, \Theta_{g}\right)_{N}=c_{g}(g, g)_{N}
$$

Thus, the evaluation of $L(g \otimes \chi, 1)$ boils down to calculating the coefficient $c_{g}$ of $g$ in the spectral decomposition (7) of the kernel function, which is accomplished by computing the Fourier coefficients of the kernel in terms of CM points on a suitable quaternion algebra.

Thus let $B$ denote the quaternion algebra over $\mathbf{Q}$ ramified precisely at $N$ and infinity, and let $R \subset B$ denote a maximal order. Recall also the notation introduced in Section 6, and let $X$ denote the set of CM points of conductor 1 and level $U=\hat{R}^{\times}$. (These are the 'special points' of discriminant $D$ in [Gro87].) Then Gross shows that $X$ admits an action of the group $\operatorname{Pic}\left(O_{K}\right)$, and that this action is both simple as well as transitive, as in section 6 above. We let $P$ denote any fixed point in $X$, and define a CM divisor by

$$
y=\sum_{\sigma \in \operatorname{Pic}\left(O_{K}\right)} \chi^{-1}(\sigma) P^{\sigma}
$$

Furthermore, Gross defines an intersection pairing $(\bullet, \bullet)$ on the space of CM divisors as follows. Each point $P \in C$ determines an oriented maximal order $R$ of $B$ as described in section 6 , and we put

$$
\left(P, P^{\prime}\right)=\delta\left(P, P^{\prime}\right)
$$

where $\delta\left(P, P^{\prime}\right)=0$ unless the orders $R, R^{\prime}$ determined by $P, P^{\prime}$ are conjugate in $B$. In the latter case, we put $\delta\left(P, P^{\prime}\right)=w$, where $w$ is the order of the finite group $R^{\times}$. We extend to pairing to CM divisors (which are just finite linear combinations of points) by linearity in the first variable, and skew-linearity in the second. Putting $S(U)=B^{\times} \backslash \hat{B} / \hat{\mathbf{Q}}^{\times} \hat{R}^{\times}$, it is clear that the pairing defined above is in fact a pairing on $S(U) \times S(U)$ (since $S(U)$ is just the set 
of conjugacy classes of oriented maximal orders) and that the pairing on CM points factors through the evident map $C \rightarrow S(U)$.

Finally, it is not hard to see that the space $S(U)$ inherits an action of the Hecke operators $T_{n}$, for all integers $n$.

With this notation, the basic result is the following

Proposition 7.2 (Gross) Write $\Theta=\sum b_{n} q^{n}$. Then the coefficients $b_{n}$ are given by

$$
b_{n}=\frac{\left(y, T_{n} y\right)}{u^{2} \sqrt{D}}
$$

where $y$ and the pairing $(\bullet, \bullet)$ are as described above, and $u=\# O_{K}^{\times} / 2$.

With all this in hand, it is now easy to prove a Gross-Zagier formula. Indeed, we want to compute the coefficient of the newform $g$ in the spectral decomposition of $\Theta$. In other words, we want to pick off the projection $\Theta_{g}$ of $\Theta$ to the space where $T_{n}$ acts via $a_{n}=a_{n}(g)$. It is not hard to see that this projection has coefficients $b_{n}^{\prime}$ given by

$$
b_{n}^{\prime}=\frac{\left(y_{g}, T_{n} y_{g}\right)}{u^{2} \sqrt{D}}
$$

where $y_{g}$ denotes the projection of the CM divisor $y$ to the subspace of $\mathbf{C}[S(U)]$ where the Hecke algebra $\mathbf{T}_{B}$ (defined by action of the $T_{i}$ on automorphic forms of level $\hat{R}^{\times}$on $B$ ) acts via the character given by the Jacquet-Langlands correspondent of $g$. In particular, the first coefficient of the projection $\Theta_{g}$ is given by

$$
b_{1}\left(\Theta_{g}\right)=\frac{\left(y_{g}, y_{g}\right)}{u^{2} \sqrt{D}} .
$$

Writing $\Theta_{g}=c_{g} \cdot g$ as in the discussion following (7), we see that $u^{2} \sqrt{D} \cdot\left(y_{g}, y_{g}\right)=$ $c_{1}\left(\Theta_{g}\right)=c_{g}$, since $g$ was normalized to have first Fourier coefficient 1 . Combining this with (8), we get the desired formula:

$$
\frac{L(g \otimes \chi, 1)}{(g, g)_{N}}=\frac{\left(y_{g}, y_{g}\right)}{u^{2} \sqrt{D}}
$$

To compare with Waldspurger's theorem, we can rewrite this in terms of test vectors and torus integrals. To do this, we will need to give an adelic description of the intersection pairing, following [Zhao1a], Section 4.1. The argument is in some sense entirely formal, but it is 
nevertheless instructive to work through the details, since this is in fact what occurs in the general case treated in Zhang's article. And since the construction is completely general, we will revert for the moment to the case of general $F$ and $K$ and $\pi$, as described in the introduction.

\section{The intersection pairing revisited}

Recall that the set of CM points of level $U$ is defined to be the coset space $C=T(F) \backslash G^{\prime}\left(\mathbf{A}_{f}\right) / U$, where $G^{\prime}=B^{\times} / F^{\times}$, and $U$ is some open compact subgroup of $G^{\prime}\left(\mathbf{A}_{f}\right)$. (In Gross' situation, we will take $U$ to denote the image of $R\left(\mathbf{A}_{f}\right)^{\times}$, for a fixed maximal order $R$.) Let $S(U)=G^{\prime}(F) \backslash \hat{G}_{f}^{\prime} / U$, where we have simply written $\hat{G}_{f}^{\prime}$ in place of $G^{\prime}\left(\mathbf{A}_{f}\right)$.

Let $m$ denote the characteristic function of $U \subset \hat{G}_{f}^{\prime}$, and consider the kernel function $k(x, y)$ defined on $S(U) \times S(U)$ by

$$
k(x, y)=\sum_{\gamma \in G^{\prime}(F)} m\left(x^{-1} \gamma y\right) .
$$

This sum is actually finite. Indeed, if $(x, y) \in \hat{G}_{f}^{\prime} \times \hat{G}_{f}^{\prime}$, then $m\left(x^{-1} \gamma y\right)$ is nonzero only for those $\gamma$ such that $\gamma \in x U y^{-1}$. But $x U y^{-1}$ is compact in $\hat{G}_{f}^{\prime}$, and $G^{\prime}\left(F_{\infty}\right)$ is compact already, so $G^{\prime}(F) \cap x U y^{-1}$ injects into the compact set $x U y^{-1} \times G^{\prime}\left(F_{\infty}\right) \subset G^{\prime}(\mathbf{A})$. Now $G^{\prime}(F)$ being discrete in $G^{\prime}(\mathbf{A})$, it follows that $G^{\prime}(F) \cap x U y^{-1}$ is finite.

Now let $p_{1}, p_{2} \in S(U)$, and let $\xi_{1}, \xi_{2}$ denote the characteristic functions of the corresponding double cosets. Define the intersection pairing via

$$
\left(p_{1}, p_{2}\right)=\left(\xi_{1}, \xi_{2}\right)=\int_{S(U)^{2}} \xi_{1}(x) k(x, y) \overline{\xi_{2}(y)} d y d x .
$$

Here the measure on $S(U)$ is induced from a left- $G^{\prime}(F)$-invariant measure on $\hat{G}_{f}^{\prime}$, normalized so that $U$ gets volume one. To calculate this pairing, observe that $\xi$ is supported on $G^{\prime}(F) g_{1} U$, while $\xi_{2}$ is supported on $G^{\prime}(F) g_{2} U$. It follows directly from the definitions that the kernel is constant on $G(F) g_{1} U \times G(F) g_{2} U$. Indeed, if the elements $g_{i}$ are in different cosets, the kernel is just zero on this set. If on the other hand the $g_{i}$ are in the same coset, put $w=G^{\prime}(F) \cap g_{1} U g_{1}^{-1}=G^{\prime}(F) \cap g_{2} U g_{2}^{-1}$ as before. Then the kernel takes the value $w$ on $G(F) g_{1} U \times G(F) g_{2} U$.

We can transfer the pairing to the set of CM points via the evident map $C \rightarrow S(U)$, as follows. Let $k^{*}(x, y)$ denote the pullback of $k(x, y)$ to $C \times C$. Let $P_{1}, P_{2}$ denote CM points, 
and let $\xi_{1}$ and $\xi_{2}$ denote the characteristic functions of the corresponding double cosets in $C=T(F) \backslash G^{\prime}\left(\mathbf{A}_{f}\right) / U$. The measure on $C$ is understood to be induced from a measure on $\hat{G}_{f}^{\prime}$ such that $U$ gets volume 1 . Then we can define the pairing by an integral over $C \times C$ as above, and we find that $\left(P_{1}, P_{2}\right)$ is zero unless the $P_{i}$ project to the same element in $S(U)$, in which case the pairing has value $w / u^{2}$, where $u$ denotes the cardinality of the set $T(F) \cap U$. Indeed, the volume of any coset $T(F) g U$ in $C$ is $1 / u$.

ExAmple 7.3 Consider the special case of $F=\mathbf{Q}$, and $N, D$ prime. Then we have recovered Gross' pairing, including the fudge factor $1 / u^{2}$ which appears in the final formulae.

For later use, we want to consider the spectral decomposition of the kernel function $k(x, y)$, as in [Zhao1a], Lemma 4.4.2. But to state the result, we first need some notation. So recall that $G^{\prime}$ is compact at infinity by hypothesis. Then $V=U \cdot G^{\prime}(\mathbf{R})$ is an open compact subgroup of $\hat{G}^{\prime}$. Recall also that we are interested in representations $\pi$ of $G L_{2}$ of parallel weight $(2, \ldots 2)$, which correspond via Jacquet-Langlands to representations $\pi^{\prime}$ on $G^{\prime}$ whose infinite component is trivial. With this in mind, let $\psi_{1}, \psi_{2}, \ldots, \psi_{r}$ denote an orthonormal basis for the space of automorphic forms on $G^{\prime}$ that are fixed by the subgroup $V$. We may assume that the $\psi_{i}$ are eigenvectors for the good Hecke operators. Since $G^{\prime}(\mathbf{R}) \subset V$, we may view any such $\psi_{i}$ as a function on the set $S(U)=G^{\prime}(F) \backslash \hat{G}_{f}^{\prime} / U$. Then the basic result is as follows.

Proposition 7.4 (Zhang) The kernel function $k(x, y)$ has the spectral decomposition

$$
k(x, y)=\sum_{i} \psi_{i}(x) \bar{\psi}_{i}(y)
$$

Proof This is easy. Indeed, if $\psi$ is any function on $S(U)$, then we have

$$
\int_{G^{\prime}(F) \backslash \hat{G}_{f}^{\prime}} \psi(y) k(x, y) d y=\int_{\hat{G}_{f}^{\prime}} \psi(y) m\left(x^{-1} y\right) d y,
$$

where $m$ denotes the characteristic function of the compact set $U$. Since $U$ has volume 1 , we get $\int_{G^{\prime}(F) \backslash \hat{G}_{f}^{\prime}} \psi(y) k(x, y) d y=\psi(x)$. Since $S(U)$ is finite, we have $k(x, y)=\sum c_{i j} \psi_{i}(x) \bar{\psi}_{j}(y)$, for some $c_{i j}$, and so the statement of the proposition follows.

Remark 7.5 The basis vectors $\psi_{i}$ above will not, in general, diagonalize the Hecke operators, as there will be some contribution from oldforms. In particular, a given eigenspace for the Hecke operators will contain several vectors $\psi_{i}$. 
Finally, we want to mention the action of the Hecke operators on the CM cycles. Given a function $\phi$ on $T(F) \backslash \hat{G}_{f}^{\prime} / U$, we define a Hecke operator $T_{v}$ for each place $v$ such that $B_{v}$ is split and $U_{v}$ is maximal as follows:

$$
T_{v} \phi(x)=\int_{H_{v}} \phi\left(x h_{v}\right) d h_{v},
$$

where $H_{v}$ is the set of matrices $h_{v}$ in $B_{v}$ such that $\operatorname{det}\left(h_{v}\right)$ is a uniformizer in $F_{v}$. Here the measure on $H_{v}$ is such that the maximal compact $U_{v}$ gets measure 1. One may check that the operator $T_{v}$ is self-adjoint with respect to the $L^{2}$ norm, so that $\left(T_{a} \phi, \phi^{\prime}\right)=\left(\phi, T_{a} \phi^{\prime}\right)$, provided that the functions $\phi$ and $\phi^{\prime}$ have compact support so that the $L^{2}$ integral makes sense. We can of course make a similar definition for functions on $S(U)=G^{\prime}(F) \backslash \hat{G}_{f}^{\prime} / U$.

\section{Gross' formula: adelic version}

We now go back to Gross' situation, where $F=\mathbf{Q}$, and $N, D$ are prime. The goal is to rewrite the special value formula (13) as a torus integral, as in Waldspurger's theorem. Obviously, we must compute the divisor $y$ defined in (10), as well as the projection to the appropriate Hecke eigenspace. The first question is rather easy. Indeed, consider the image of $T(\mathbf{Q}) \backslash \hat{T}=$ $\operatorname{Pic}\left(O_{K}\right)$ inside the set $C=T(\mathbf{Q}) \backslash G^{\prime}\left(\mathbf{A}_{f}\right) / U$. Each class $\sigma \in \operatorname{Pic}\left(O_{K}\right)$ defines a CM point $\xi_{\sigma} \in C$. We may therefore form the CM divisor

$$
y=\sum \chi(\sigma) \xi_{\sigma}
$$

Notice that there is no need to specify a base point with this formulation.

Next we need to make sense of the projection to the Hecke eigenspace of interest. To do this, it will be convenient to identify the CM point $\xi_{\sigma}$ with the characteristic function of the corresponding double coset in $C$. By definition of the intersection pairing, we have

$$
(y, y)=\int_{C^{2}} \xi_{y}(u) k^{*}(u, v) \bar{\xi}_{y}(v) d u d v .
$$

In view of the spectral decomposition of the kernel in Proposition 7.4, the integral above may be rewritten as

$$
(y, y)=\sum \int_{C} \xi_{y}(u) \psi_{i}(u) \bar{\psi}_{i}(v) \bar{\xi}_{y}(v) d u d v,
$$

where the sum is taken over an orthonormal basis for the space of cuspforms on $S(U)$. In the situation where $F=\mathbf{Q}$ and the level $N$ is prime, there are in fact no oldforms, so we 
may take the basis vectors $\psi_{i}$ to be Jacquet-Langlands newforms, each belonging to a distinct eigenspace for the Hecke algebra. We may assume by renumbering that our original representation $\pi^{\prime}$ corresponds to the vector $\psi=\psi_{1}$.

Thus the pairing decomposes as

$$
(y, y)=\sum_{i}\left(\int_{C} \xi_{y}(u) \psi_{i}(u) d u\right)\left(\int_{C} \bar{\psi}_{i}(v) \bar{\xi}_{y}(v) d v\right) .
$$

By self-adjointness of the Hecke operators, we have

$$
\int_{C} T_{a} \xi_{y}(u) \psi_{i}(u) d u=\int_{C} \xi_{y}(u) T_{a} \psi_{i}(u) d u
$$

for any a prime to the level $N$. Since $N$ is prime, strong multiplicity one implies that, if $y_{\pi^{\prime}}$ denotes the projection of $y$ to the eigenspace corresponding to $\pi^{\prime}$, then we have the formula

$$
\left(y_{\pi^{\prime}}, y_{\pi^{\prime}}\right)=\left(\int_{C} \xi_{y}(u) \psi(u) d u\right)\left(\int_{C} \bar{\xi}_{y}(v) \bar{\psi}(v) d v\right) .
$$

But it is easy to see that the integrals above are precisely torus integrals of the test vector $\psi$, since the function $\xi_{y}$ is supported on the image of the torus $\hat{T}$. Indeed, we have

$\ell_{\chi}(\psi)=\int_{C} \xi_{y}(u) \psi(u) d u=1 / u \sum_{t \in T(F) \backslash \hat{T} / \hat{O}_{K}} \chi^{-1}(t) \psi(t)=\int_{T(F) \backslash \hat{T} / T\left(\hat{O}_{K}\right)} \chi^{-1}(t) \psi(T)$,

where the measure on $\hat{T}$ is such that $T\left(\hat{O}_{K}\right)$ gets measure 1 .

Thus we may rewrite Gross' formula in the following form:

$$
\frac{L(g \otimes \chi, 1)}{(g, g)_{N}}=\frac{|\ell(\psi)|^{2}}{\sqrt{D}}
$$

where $\ell$ is the torus integral with respect to a Haar measure giving $T\left(\hat{O}_{K}\right)$ volume 1 , and $\psi$ is a Jacquet-Langlands newform, normalized to have $L^{2}$ norm 1 , with respect to a Haar measure on $\hat{G}^{\prime}$. Here the measure on $\hat{G}^{\prime}$ is normalized so that a maximal compact $U \subset \hat{G}^{\prime}$ has volume equal 1 .

The work of Zhang

We now want to describe the formula of [Zhao1a]. We therefore return to the general situation, keeping the notation fixed in Section 2. Thus we want to study the special value 
$L(\pi \otimes \chi, 1 / 2)$, and the starting point is once again the expression of the L-series as a Rankin $\mathrm{L}$-function. The basic strategy is the same as in the discussion above, but with some important improvements. We will attempt here to at least explain the statement of the final formula, if not all the new ideas introduced in Zhang's proof.

\section{Quasi-newforms on $G L_{2}$}

As before, one begins with a theta kernel $\Theta_{\chi}$, which (under the present hypotheses) is an automorphic form of weight $(2, \ldots, 2)$ on $G L_{2}$, and which satisfies the equation

$$
L(\pi \otimes \chi, 1 / 2)=\left(\phi, \Theta_{\chi}\right)
$$

where $\phi$ denotes the Atkin-Lehner newform of level $N$ associated to $\pi$ as described in section 3. Here the form $\Theta_{\chi}$ is of level $N D$, where $D=d c^{2}, d$ is the discriminant of $K / F$, and $c$ is the conductor of $\chi$.

Note that there are various normalizations already implicit in the above formula. Specifically, the formula holds with $\phi$ normalized as in section 3, and the inner product is taken with respect to the $L^{2}$ metric on $G L_{2}(F) \hat{F}^{\times} \backslash G L_{2}(\hat{F})$. The Haar measure on the latter is fixed so that the open compact subgroup $U_{0}(N D) K_{\infty}$ gets measure 1 , where $K_{\infty}$ is a product of the various maximal compact subgroups at primes dividing infinity. The detailed construction of the kernel $\Theta_{\chi}$ is given in Chapter 2 and section 3.1 of [Zhaora].

If we were to follow the classical argument exactly, the next step would be to compute the trace of the theta kernel down to level $N$. This appears to be rather difficult, since the extra level $D=d c^{2}$ is large when $c \neq 1$. Thus Zhang works directly with the kernel function at level $N D$, as follows. Let $\pi_{1}, \pi_{2}, \ldots, \pi_{r}$ be an enumeration of the finitely many cuspidal automorphic representations of weight $(2, \ldots, 2)$ occurring at levels dividing $N D$. Then $\pi=\pi_{j}$ for some $j$, say $j=1$, and we may decompose $\Theta_{\chi}$ in a manner analogous to (7), as

$$
\Theta_{\chi}=\text { Eisenstein part }+\sum \phi_{i}
$$

where each $\phi_{i}$ is a form in the Hecke eigenspace corresponding to the representation $\pi_{i}$. Then one has

$$
L(\pi \otimes \chi, 1 / 2)=\left(\phi, \Theta_{\chi}\right)=\left(\phi, \phi_{\pi}\right),
$$

where $\phi_{\pi}=\phi_{1}$ is the term in (18) corresponding to the fixed representation $\pi$. 
However, since we are working at level $N D$, and $\phi$ has level $N$, it is no longer true that $\phi_{\pi}$ is simply a scalar multiple of the newform $\phi$. Indeed, the best we can say is that $\phi_{\pi}$ is some linear combination

$$
\phi_{\pi}=\sum c_{a} \phi_{a}
$$

where the sum is taken over ideals $a$ dividing $D$, and $\phi_{a}$ is as in Casselman's theorem. It is evident from this that, even if one could compute the Fourier coefficients of $\Theta_{\chi}$ in terms of CM cycles, a simple argument as in (12) cannot be made to work, since one has no evident normalization of the form $\phi_{\pi}$. Note here that it is crucial to the argument following (12) that the newform $g$ was normalized to have first Fourier coefficient 1.

In view of the considerations above, we are led to normalize the vector $\phi_{\pi}$ in some convenient way. In other words, we are looking for some distinguished vector on the line $L$ spanned by the vector $\phi_{\pi}$.

Definition 7.6 Let $\mathcal{S}$ denote the space of cuspforms of level $N D$. If $L$ is any line in $\mathcal{S}$, then the quasi-newform $\phi^{\#}=\phi_{L}^{\#}$ associated to $\pi$ on the line $L$ is the orthogonal projection of the Atkin-Lehner newform $\phi$ on $L$.

Remark 7.7 The definition assumes that $\phi$ has already been normalized. This already occurs in the formula (17).

Remark 7.8 By definition, there is a quasi-newform on each line in the ambient space $\mathcal{S}$. Thus for the definition to be useful, one needs to specify the line $L$. Note that the quasinewform attached to $L$ is zero if $L$ is orthogonal to the Atkin-Lehner newform $\phi$.

Remark 7.9 Let the line $L$ be given. Suppose that $L$ is not orthogonal to $\phi$ so that $\phi_{L}^{\#} \neq 0$. Then if $v$ is any vector on $L$, we will have $v=c \phi_{L}^{\#}$, for some scalar $c$. For simplicity, we assume that $c$ is real, which will be the case in our applications. One then has

$$
(\phi, v)=\left(\phi, c \phi_{L}^{\#}\right)=c\left(\phi, \phi_{L}^{\#}\right)=c\left(\phi_{L}^{\#}, \phi_{L}^{\#}\right)
$$

since $\phi_{L}^{\#}$ is the orthogonal projection of $\phi$ on $L$.

Let us apply this to the formula (19) with $L$ taken to be the line spanned by $\phi_{\pi}$. Then (19) implies that $L(\pi \otimes \chi, 1 / 2)=0$ if and only if $L$ is orthogonal to $\phi$, or $\phi_{\pi}$ is zero. If this not the case, then we may put $v=\phi_{\pi}=c_{\pi} \phi_{L}^{\#}$, and this gives the following key formula:

$$
L(\pi \otimes \chi, 1 / 2)=\left(\phi, \phi_{\pi}\right)=c_{\pi}\left(\phi_{L}^{\#}, \phi_{L}^{\#}\right) .
$$


We are therefore led to compute the line $L$ spanned by the vector $\phi_{\pi}$, and to determine the corresponding quasi-newform $\phi_{L}^{\#}$. The former problem has been solved by Zhang, as we now explain.

Thus recall that the form $\phi_{\pi}$ is by definition the projection of the kernel $\Theta_{\chi}$ to the eigenspace (for the good Hecke operators) corresponding to the representation $\pi$. According to Casselmans' theorem, a basis for this eigenspace is given by the functions $\phi_{a}$, where $a$ runs over the ideals dividing $D$. Thus we have $\phi_{\pi}=\sum x_{a} \phi_{a}$, and we must compute the coefficient $x_{a}$ for each $a$. Note here that the vectors $\phi_{a}$ are not orthonormal, or even orthogonal.

In practice, it is much easier to compute the inner products $\left(\phi_{\pi}, \phi_{a}\right)$, thereby determining the orthogonal complement to our line $L$, rather than $L$ itself. To state the result, we need some notation. Recall that the character $\chi$ is anticyclotomic. For each prime $v$ of $F$ which is ramified in $K$, one sees therefore that the local character $\chi_{v}$ is quadratic. It follows that $\chi_{v}$ is the composition of some unramified character $v$ of $F_{v}$ with the local norm from $K_{v}$ to $F_{v}$.

Now, for any place $v$ dividing $N D$, define a function $v^{*}(v)$ as follows. If $v$ is ramified in $K$, we put $v^{*}(v)=v\left(\pi_{v}\right)$ where $\pi_{v}$ is a uniformizer of $F_{v}$, and $v$ is the local character defined above. If $v$ is unramified in $K$, we put $v^{*}(v)=0$. We can extend the function $v^{*}$ to the set of ideals dividing $N D$ by multiplicativity.

Proposition 7.10 (Zhang) Let $\mathcal{S}_{\pi}$ denote the linear span of the vectors $\phi_{a}$, as a runs over divisors of ND. Let $\phi_{\pi} \in \mathcal{S}$ denote the projection of $\Theta_{\chi}$ to $S_{\pi}$. Then:

- If $L(\pi \otimes \chi, 1 / 2) \neq 0$, then $\phi_{\pi} \neq 0$, and the line $L$ spanned by $\phi_{\pi}$ is the orthogonal complement in $S_{\pi}$ of the hyperplane given by $\sum_{a} c_{a} \phi_{a}$, where

$$
\sum c_{a} v^{*}(a)=0
$$

- If $L(\pi \otimes \chi, 1 / 2)=0$, then $\phi_{\pi}=0$.

The proof of this is a somewhat elaborate computation, based on the precise definition and normalization of the kernel function $\Theta_{\chi}$, which enables one to calculate $\left(\phi_{a}, \Theta_{\chi}\right)$ for any $a$. The details may be found in Section 3.1 of [Zhao1a]. Note also here that $L$ is not orthogonal to the Atkin-Lehner newform, so the quasi-newform $\phi_{L}^{\#}$ is non-zero.

Let $L$ denote the line defined in the first part of Proposition 7.10. Then $\phi^{\#}=\phi_{L}^{\#}$ will denote the (nonzero) quasi-newform associated to this $L$. We put $c_{\pi}=0$ if $L(\pi \otimes \chi)=0$, 
and we define it by equation (20) if not. Then, with this convention, it is clear that (20) holds in general. Indeed, the identity

$$
\phi_{\pi}=c_{\pi} \phi^{\#}
$$

holds in general as well.

\section{Toric newforms on the quaternion algebra $B$}

The next step in the argument is to calculate the Fourier (or Whittaker) coefficients of the kernel function $\Theta_{\chi}$ in terms of CM cycles on the quaternion algebra $B$, and then to express the final formula in terms of a Waldspurger functional on some suitable test vector. Again, the details are somewhat involved, so we will limit ourselves to explaining the statements and results that are relevant to our purposes. The details may be found in Chapter 4 of [Zhaora]. We point out here that the results in [Zhaora] include a general construction of geometric intersection pairings on CM cycles, and in fact yield a very beautiful local version of the Gross-Zagier formula, neither of which we will attempt to discuss here. We will just describe Zhang's construction of toric newforms, which are the analog in his set-up of the test vectors described in Section 6 above. The two notions are related, but are not equivalent.

Recall that we have fixed an embedding $K \rightarrow B$. Let $O_{K}$ denote the ring of integers in $K$. Then we let $R \subset B$ denote an order defined by

$$
R_{v}=O_{K, v}+O_{K, v} \lambda_{v}, \quad \lambda_{v}=\varpi_{v}^{m} \epsilon_{v}
$$

where $\lambda_{v}, \varpi_{v}$, and $\epsilon_{v}$ satisfy the following conditions:

1. $\epsilon_{v} x=\bar{x} \epsilon_{v}$, for each $x \in K_{v}$. Here $\bar{x}$ denotes the conjugate of $x$ over $F_{v}$.

2. $\epsilon_{v}^{2} \in K_{v}$ has valuation 0 unless $B$ is nonsplit and $K / F$ is unramified, in which case it has valuation 1 .

3. $\lambda_{v}=\varpi_{v}^{m} \epsilon_{v}$, where $\varpi_{v} \in O_{K, v}$ is a local uniformizer in $K_{v}$ if $K_{v}$ is a field, and $\varpi_{v}$ is a local uniformizer of one factor of $v$ in $K$ if $v$ is split.

4. $m=\operatorname{ord}_{v}(c)$, where $c$ is the conductor of $\chi$. 
Note that $R_{v}$ is maximal for almost every $v$. Note also that $\lambda$ generates a two-sided ideal in $R$ by virtue of conditions 1 and 3 above. By condition 4 , there is an identification $\hat{R} / \lambda \hat{R} \cong$ $\hat{O}_{K} / c \hat{O}_{K}$. Since $\chi$ is a character of conductor $c$, it defines a character of $\left(\hat{O}_{K} / c \hat{O}_{K}\right)^{\times}$, and we thereby deduce an extension of $\chi$ to $\hat{R}^{\times}$. By abuse of notation, we will continue to denote the extended character by $\chi$. Note however that, as a character of $\hat{R}^{\times}, \chi$ is trivial at all places $v \nmid c$, since $\chi_{v}$ is trivial on the units of $K_{v}$ at places away from the conductor.

Finally, we define a subgroup $\Delta \subset \hat{B}^{\times}$as follows. If $v$ is unramified in $K$, we put

$$
\Delta_{v}=F_{v}^{\times} R_{v}^{\times} .
$$

If $v$ is ramified in $K$, we put

$$
\Delta_{v}=K_{v}^{\times} R_{v}^{\times} .
$$

This definition makes sense, since $K_{v}$ is normalized by $R_{v}$, so the product in the definition above is indeed a group. Furthermore, one checks that $\chi$, which is defined on both $K_{v}$ and $R_{v}$, extends in an obvious way to $\Delta$. We write $\chi_{\Delta}$ for this extended character.

With this definition, Zhang gives the following definition and existence result.

Definition 7.11 ([Zha01A ], Section 2.3) Let the group $\Delta$ be as above, and let $\chi_{\Delta}$ denote the character of $\Delta$ constructed above. A vector $\phi_{\chi}$ in $\pi^{\prime}$ is called a toric newform with character $\chi$ if

- $\phi_{\chi}$ is an eigenform for the Hecke operator $T_{v}$ at all places $v$ away from $N D$, and

- The action of $\Delta$ on $\phi_{\chi}$ is given by the character $\chi_{\Delta}$.

Proposition 7.12 There exists a unique line in the representation $\pi^{\prime}$ where $\Delta$ acts via the character $\chi_{\Delta}$. Thus nonzero toric newforms exist.

Remark 7.13 The existence and uniqueness of the toric newvector $\psi_{\chi}$ may be checked locally. As we have already remarked, $\chi_{\Delta}$ is trivial at all primes away from $D$. Thus if $\psi=\otimes \psi_{v}$ denotes the newform attached to the representation $\pi^{\prime}$ in Section 4 , we may simply take $\phi_{\chi, v}=\psi_{v}$ for all $v$ away from $D$. This already gives the Hecke eigenvector property.

As for the places $v$ dividing $D$, one has to replace $\psi_{v}$ with a local vector $\phi_{\chi, v}$ satisfying the appropriate transformation property under $\Delta_{v}$. This is similar to the techniques from [GP91]. Note, however, that optimal embeddings do not appear directly in the present setup. We refer the reader to Chapter 2 of [Zhao1a] for the details. 
Remark 7.14 It may be of interest to explicate the connection between the toric newform and the test vector of Gross-Prasad. It is easy to see that the two notions are the same at primes away from the conductor of $\chi$, so the we need only consider primes $v$ where $\chi$ is ramified. While the general relationship is complicated, we can make a simple statement at primes $v \mid c(\chi)$ which are inert in $K$.

Thus, let $v$ denote such a prime. Then the local representation $\pi_{v}=\pi_{v}^{\prime}$ decomposes under the action of the torus $T$ as the sum of one-dimensional invariant subspaces (see [Tun83]). The Gross-Prasad local test vector has the property that it projects nontrivially on to any line where the action of $T$ is via a character of conductor dividing that of $\chi$. Zhang's test vector, on the other hand, lies on the line where $T$ acts via the fixed character $\chi$ of interest.

\section{The final formula}

We are now almost ready to state Theorem 1.3.2 of [Zhao1a], which gives a formula for $L(\pi \otimes$ $\chi, 1 / 2)$. But first we need to specify precisely the groups we work with, and the normalizations of the measures and vectors which will appear.

Let $G=G L_{2}(F) / F^{\times}$. Then we normalize the Haar measure on $G(\mathbf{A})$ by requiring that $U_{0}(N D)$ get measure 1 . On the quaternion side, we let $G^{\prime}=B^{\times} / F^{\times}$, and let $U \subset \hat{R}$ denote the kernel of $\chi$. We fix the measure on $\hat{G}^{\prime}$ so that $\Delta$ gets measure 1 . Here $R$ denotes the order constructed in Section 7. Note that in each case, the measure actually depends on the conductor of $\chi$. Finally, we fix a Haar measure on $\hat{T}=\hat{K}^{\times} / \hat{F}^{\times}$by requiring that the maximal compact subgroup gets measure 1 . This of course is independent of $\chi$.

REMARK 7.15 The subgroup $U$ giving the level is not simply the image of $\hat{R}^{\times}$. Rather, it is the subgroup of $\hat{R}^{\times}$corresponding to the kernel of $\chi$. This will be important in understanding the statements.

Theorem $7.16(\mathrm{ZHANG})$ We have $L(\pi \otimes \chi, 1 / 2)=0$ if and only if $\ell\left(\phi_{\chi}\right) \neq 0$. If $L(\pi \otimes$ $\chi, 1 / 2) \neq 0$, then we have the formula

$$
L(\pi \otimes \chi, 1 / 2)=\frac{2^{n}\left|\phi^{\#}\right|^{2}}{c^{\#} \sqrt{D_{K / F}}} \cdot\left(\frac{\int_{F^{\times} \backslash \hat{T}} \chi^{-1}(t) \phi_{\chi}(t) d t}{\left|\phi_{\chi}\right|}\right)^{2}
$$

Here $n$ denotes the degree of $F / \mathbf{Q}, D_{K / F}$ denotes the absolute norm of the discriminant of $K / F$, and $c^{\#}$ is the first Whittaker coefficient of the quasi-newform $\phi^{\#}$ (the precise definition is 
given below). The norms are understood to be the $L^{2}$ norms relative to the measures specified above.

Remark 7.17 The number $c^{\#}$ is missing from the statement in [Zhao1a]. Its appearance is explained below.

Needless to say, we are not in a position to say anything substantial about the proof of this theorem. Broadly, it follows the lines sketched in the discussion of Gross' formula. We have already indicated above how the fact that Zhang works at level $N D$ leads to the appearance of the quasi-newform $\phi^{\#}$, rather than the newform $\phi$. Let us therefore briefly indicate how the toric newform makes an appearance on the quaternion algebra side of the question. In the process, we will also explain the factor $c^{\#}$.

As we have remarked several times, the basic point is to relate the Whittaker coefficients of the theta kernel to the values of an intersection pairing. Thus, recall that the theta kernel on $G L_{2}$ is determined by its Whittaker coefficients

$$
W\left(\left(\begin{array}{ll}
a & 0 \\
0 & 1
\end{array}\right) \cdot g_{\infty}\right),
$$

where $a$ runs over the finite ideles of $F$, and $g_{\infty} \in G_{\infty}=G(F \otimes \mathbf{R})$. To define the Whittaker functions, we assume fixed a nontrivial additive character $\eta$ of $\hat{F} / F$. We let $\delta$ denote the conductor of $\eta$.

We want to express the Whittaker coefficient above, which is a function of $a$ and the parameter $g_{\infty}$, as the value of a pairing on CM cycles. Let $y_{\chi}$ denote the CM cycle given by the following compactly supported function $y_{\chi}=\prod y_{\chi, v}$ on $C$, where $y_{\chi, v}$ is supported on $T\left(F_{v}\right) R_{v}^{\times}$and satisfies

$$
y_{\chi, v}(t u)=\chi(t) \chi(u), t \in T\left(F_{v}\right), u \in \Delta_{v} .
$$

where $G_{c}=T \backslash \hat{T} / \hat{O}_{c}$ as before, and $\xi_{\sigma}$ is the corresponding element of $C$. For each $g_{\infty} \in G_{\infty}$, we define an intersection pairing $(\bullet, \bullet)\left(g_{\infty}\right)$ on the space of CM cycles as in Gross' theorem, where the multiplicity function $m=m_{g_{\infty}}$ depends on $g_{\infty}$. Indeed, we define

$$
m_{g_{\infty}}(x)=2^{n} W_{\infty}\left(g_{\infty}\right) m(x), x \in G^{\prime}(F) \backslash \hat{G}_{f}^{\prime}
$$

where $W_{\infty}$ is a standard Whittaker function for the weight 2 discrete series on $G_{\infty}$, and $m$ is the characteristic function of $U$. (See [Zhao1a], Eq. 4.4.2.) With this definition, Zhang 
proves that the Whittaker coefficients of $\Theta_{\chi}$ satisfy

$$
W\left(\left(\begin{array}{cc}
a \delta^{-1} & 0 \\
0 & 1
\end{array}\right) \cdot \epsilon g_{\infty}\right)=\frac{|a|\left(T_{a} y_{\chi}, y_{\chi}\right)\left(g_{\infty}\right)}{\sqrt{D_{K / F}}}
$$

for all ideles $a$ with component 1 at places dividing $N D$. Here $\epsilon=\left(\begin{array}{cc}1 & 0 \\ 0 & -1\end{array}\right)$.

Let $\phi_{\pi}$ denote the projection of $\Theta_{\chi}$ to the $\pi$-isotypic part. Then we have

$$
\phi_{\pi}=c_{\pi} \phi^{\#}
$$

for the quasi-newform $\phi^{\#}$, with the conventions of (21). We now examine what this means in terms of the intersection pairings, as in the argument following (12). According to the strong multiplicity one theorem, we can pick off the the $\pi$-isotypic component of $\Theta_{\chi}$ by means of the good Hecke operators. In other words, there exists some polynomial $t$ in the good Hecke operators $T_{a}$ such that if $\Theta_{\chi}=\sum \phi_{i}$ as in (18), then $t \Theta_{\chi}=\phi_{\pi}$ is the $\pi$-isotypic part. Thus we can compute the first Whittaker coefficient of $\phi_{\pi}$, using the formulae (1) for the action of Hecke operators on Whittaker coefficients:

$$
W_{\phi_{\pi}}\left(\left(\begin{array}{cc}
\delta^{-1} & 0 \\
0 & 1
\end{array}\right) \cdot \epsilon g_{\infty}\right)=\frac{\left(t y_{\chi}, y_{\chi}\right)\left(g_{\infty}\right)}{\sqrt{D_{K / F}}}
$$

On the other hand, we have $\phi_{\pi}=c_{\pi} \phi^{\#}$ by definition. So we would like to compare Whittaker coefficients, but we have to be slightly careful, since certain coefficients may a priori be zero. Define a number $c^{\#}\left(g_{\infty}\right)$ by the formula

$$
c^{\#}\left(g_{\infty}\right)=W_{\phi^{\sharp}}\left(\left(\begin{array}{cc}
\delta^{-1} & 0 \\
0 & 1
\end{array}\right) \epsilon g_{\infty}\right) .
$$

Lemma 7.18 The function $c^{\#}\left(g_{\infty}\right)$ is nonzero, and $c^{\#}=c^{\#}(1) \neq 0$.

For a proof of this lemma, we refer the reader to forthcoming work of Zhang and to his article in this volume.

With this is mind, we can write $\phi_{\pi}=c_{\pi} \phi^{\#}$ and compare Whittaker coefficients. Then we get 


$$
c_{\pi}=\frac{\left(t y_{\chi}, y_{\chi}\right)\left(g_{\infty}\right)}{c^{\#}\left(g_{\infty}\right) \sqrt{D_{K / F}}} .
$$

To evaluate $c_{\pi}$, we may take $g_{\infty}=1$. In this case, we will simply drop it from the notation. Then comparing with the equation (20), we find that

$$
\frac{L(\pi \otimes \chi, 1 / 2)}{\left(\phi^{\#}, \phi^{\#}\right)}=\frac{\left(t y_{\chi}, y_{\chi}\right)}{c^{\#} \sqrt{D_{K / F}}} .
$$

It remains to compute the pairing $\left(t y_{\chi}, y_{\chi}\right)$, and this we can compute via the spectral decomposition in Proposition 7.4. When $g_{\infty}=1$, it turns out that the kernel for the intersection pairing has the decomposition

$$
k^{*}(x, y)=2^{n} \sum_{i} \phi_{i}(x) \bar{\phi}_{i}(y),
$$

where $n$ is the degree of $F / \mathbf{Q}$, and the sum is taken over an orthonormal basis for the space of forms of level $U$. We may assume that these basis vectors are all eigenvectors for the good Hecke operators. Thus the pairing $\left(t y_{\chi}, y_{\chi}\right)$ becomes

$$
\left(t y_{\chi}, y_{\chi}\right)=2^{n} \sum\left(\int_{C} t y_{\chi}(u) \phi_{i}(u) d u\right)\left(\int_{C} \bar{\phi}_{i}(v) \bar{y}_{\chi}(v) d v\right),
$$

By the self-adjointness of the Hecke operators and the definition of the projection operator $t$, the contribution from vectors $\psi_{i}$ corresponding to eigenspaces other than $\pi^{\prime}$ are all wiped out. Here we use the fact the Jacquet-Langlands correspondence preserves the Hecke eigenvalues. The vectors that remain correspond to an orthonormal basis for the $\pi^{\prime}$-isotypic subspace of forms of level $U$, which will, in general, have dimension strictly greater than 1 . Thus suppose that $\phi_{1}, \ldots, \phi_{r}$ are an orthonormal basis for the space of $\pi^{\prime}$-isotypic forms of level $U$. We may assume that $\phi_{1}$ is the toric newvector. Then we want to show that the terms for $i=2, \ldots, r$ in (27) are all zero as well.

Consider therefore the action of the subgroup $\Delta$ on the functions $\phi_{i}$ by the usual right translation. Then $\Delta$ acts on $\phi_{\chi}=\phi_{1}$ by the character $\chi$, by definition of the toric newvector. Since $\pi^{\prime}$ is unitary, the action of $\Delta$ preserves the orthogonal complement of $\phi_{1}$, namely, the space spanned by $\phi_{2}, \ldots, \phi_{r}$. Now $\Delta$ is a totally disconnected group, so any finite dimensional complex representation of $\Delta$ factors through a finite quotient. Then the complement 
of $\phi_{1}$ breaks up as the sum of irreducible representations, all distinct from $\chi$, since the space of toric newforms is one dimensional. Since $\Delta$ acts on the function $y_{\chi}$ by the character $\chi$, it follows that the terms for $\phi_{i}$ with $i \geq 2$ are all zero.

Thus we have shown that $\left(t y_{\chi}, y_{\chi}\right)=2^{n}\left|\int_{C} y_{\chi}(u) \phi_{\chi}(u)\right|^{2}$. But it is easy to see that the quantity on the right is just a torus integral of the test vector $\phi_{\chi}$.

Remark 7.19 To conclude, we want to indicate what is not yet proven. For the purposes of Iwasawa theory and $p$-adic L-functions, it would be desirable to reformulate Zhang's theorem in terms of the fixed level structure $N$ of $\pi$. As in [BD96] and [Vato2], the desired formula should have the shape

$$
|\ell(\psi)|^{2}=C_{\chi} \frac{L(\pi \otimes \chi, 1 / 2)}{(\phi, \phi)_{N}}
$$

where $\psi$ is the Gross-Prasad test vector, $\phi$ denotes the Atkin-Lehner newform for $\pi$, and the number $C_{\chi}$ is an explicit constant in $\overline{\mathbf{Q}}$ depending on $\chi$. While it is obvious from Zhang's result that such a formula holds up to some algebraic constant, it does not seem easy to compute the number $C_{\chi}$. The main problem is determining the length of the quasi-newform in Zhang's theorem. For a discussion of this point we refer the reader to Zhang's article in this volume. We would like to point out, however, that for the purposes of the main results in [Vato2] and [BD96] and its various sequels, it is enough to know that nonvanishing of the L-function is equivalent to nonvanishing of $\ell(\psi)$, and this is true unconditionally in view of the results of Waldspurger and Gross-Prasad: see Theorem 6.4.

\section{REFERENCES}

[BD96] M. Bertolini and H. Darmon, Heegner points on Mumford-Tate curves, Invent. Math. 126 (1996), no. 3, 413-456.

[BD97] - A rigid analytic Gross-Zagier formula and arithmetic applications, Ann. of Math. (2) 146 (1997), no. 1, 111-147, With an appendix by Bas Edixhoven.

[BJ79] A. Borel and H. Jacquet, Automorphic forms and automorphic representations, Automorphic forms, representations and $L$-functions (Proc. Sympos. Pure Math., Oregon State Univ., Corvallis, Ore., 1977), Part 1, Amer. Math. Soc., 1979, With a 
supplement "On the notion of an automorphic representation" by R. P. Langlands, pp. 189-207.

[Bum97] Daniel Bump, Automorphic forms and representations, Cambridge University Press, Cambridge, 1997.

[Cas73] W. Casselman, On some results of Atkin and Lehner, Math. Ann. 201 (1973), 301314 .

[Dag96] H. Daghigh, Quaternion algebras, Ph. D. thesis, McGill University, 1996.

[GP91] B. Gross and D. Prasad, Test vectors for linear forms, Math. Ann. 291 (1991), 343-355.

[Gro] B. Gross, article in this volume.

[Gro87] - Heights and the special values of L-series, Number Theory (H. Kisilevsky and J. Labute, eds.), CMS Conference Proceedings, vol. 7, Amer. Math. Soc., 1987, pp. 115-189.

[Gro88] L Local orders, root numbers, and modular curves, Am. J. Math. 110 (1988), $1153-1182$.

[Lub94] A. Lubotzky, Discrete groups, expanding graphs and invariant measures, Birkhäuser Verlag, Basel, 1994, With an appendix by J. Rogawski.

[Maz84] B. Mazur, Modular curves and arithmetic, Proceedings of the International Congress of Mathematicians, Vol. 1, 2 (Warsaw, 1983), PWN, 1984, pp. 185-211.

[Shi76] G. Shimura, The special values of zeta functions associated with cusp forms, Comm. Pure Applied Math. 29 (1976), 783-804.

[Tun83] J. Tunnell, Local $\varepsilon$-factors and characters of $G L_{2}$, Am. J.Math. 105 (1983), 1277-1308.

[Vato2] V. Vatsal, Uniform distribution of Heegner points, Invent. Math. 148 (2002), 1-46.

[Wal85] J.-L. Waldspurger, Sur les valeurs des certaines fonctions L automorphes en leur centre de symmétrie, Compos. Math. 54 (1985), 174-242.

[Zhao1a] S. Zhang, Gross-Zagier formula for $\mathrm{gl}_{2}$, Asian J. Math. 5 (2001), no. 2. 
[Zhao1b] _ Heights of Heegner points on Shimura curves, Ann. of Math. (2) 153 (2001), no. $1,27-147$. 